\title{
Infrared deflectometry for the inspection of diffusely specular surfaces
}

DOI 10.1515/aot-2016-0051

Received September 12, 2016; accepted November 5, 2016; previously published online December 8, 2016

Abstract: Deflectometry is a full-field gradient technique that lends itself very well to testing specular surfaces. It uses the geometry of specular reflection to determine the gradient of the surface under inspection. In consequence, a necessary precondition to apply deflectometry is the presence of at least partially specular reflection. Surfaces with larger roughness have increasingly diffuse reflection characteristics, making them inaccessible to usual deflectometry. However, many industrially relevant surfaces exist that change their reflection characteristic during production and processing. An example is metal sheets that are used as car body parts. Whereas the molded but otherwise raw metal sheets show a mostly diffuse reflection without sufficient specular reflection, the final car body panels have a high specular reflectance due to the lacquering. In consequence, it would be advantageous to apply the same inspection approach both for the raw material and for the final product. To solve this challenge, specular reflection from rough surfaces can be achieved using light with a larger wavelength, as the specular reflectivity of a surface depends on the ratio of the surface roughness and the wavelength of the light applied. Wavelengths in the thermal infrared range create enough specular reflection to apply deflectometry on many visually rough metal surfaces. This contribution presents the

\footnotetext{
*Corresponding author: Jan Burke, Fraunhofer Institute of Optronics, System Technologies and Image Exploitation - Systems for Measurement, Control and Diagnosis (MRD), Karlsruhe, DE 76131, Fraunhoferstraße 1, Germany,

e-mail: Jan.Burke@IOSB.Fraunhofer.de

Sebastian Höfer: Fraunhofer Institute of Optronics, System Technologies and Image Exploitation - Systems for Measurement, Control and Diagnosis (MRD), Karlsruhe, DE 76131, Fraunhoferstraße 1, Germany

Michael Heizmann: Karlsruher Institut für Technologie (KIT) Institute of Industrial Information Technology, Hertzstraße 16, Geb. 06.35, Karlsruhe 76187, Germany
}

principles of thermal deflectometry, its special challenges, and illustrates its use with examples from the inspection of industrially produced surfaces.

Keywords: deflectometry; infrared displays; metrological instrumentation.

\section{Introduction}

This section presents the basic ingredients needed for understanding the technique of deflectometry and appreciating the rationale for extending the wavelength range into the infrared.

\subsection{Deflectometry}

When testing and/or measuring reflective objects, the well-established technique of fringe projection [1] is not available because there is not enough scattered light available to carry out measurements of the object geometry by capturing the diffuse reflection of the object surface. Ideal mirrors (which are very rare in practice) generate no scattered light at all. This means that the surface of a mirror cannot be measured directly by fringe projection. Still, the geometry of such surfaces is accessible to indirect measurements: a known reference pattern and a camera are placed in such a geometrical relationship with the object to be tested that the camera can observe the mirror image of the reference pattern 'on' the test object [2-8]. Any slopes or slope changes of the object surface will then reveal/encode themselves in displacements and distortions of the recorded reference pattern, which can readily be understood to represent the first derivatives of the test surface. This means also that both partial derivatives are needed to reconstruct the actual surface shape by integration if required [9-13]. As deflectometry has no intrinsic sensitivity to the object distance and the integration problem is therefore underdetermined, additional knowledge such as an additional point measurement or data from an additional deflectometric measurement must be used $[4,5,10]$. Another possibility to use the 
deflectometric measurement is to just evaluate the deviation of the observed reflection from the reflection that an error-free surface would show. In many cases, e.g. when only the presence of geometrical surface deviations is of interest rather than their actual geometry, a mathematical surface reconstruction and the associated calibration effort can thus be avoided.

Whether or not a 3-D surface is to be reconstructed, geometry information is required about the directions of the rays that form the image of the reference pattern; in other words, the unique relationship between coordinates on the reference screen and camera pixels must be found for each camera pixel. For objects that are specularly reflective in the visible wavelength range, this process is usually done by coding the screen pixels using a series of fringe patterns, e.g. Gray codes and/or phase shifting [14] combined with unwrapping techniques $[15,16]$. To that end, a series of images, which depicts all reference patterns of the code, is recorded with the same placement of the surface, the screen, and the camera. Provided that the measurement noise of the brightness in the recorded series is low, a position uncertainty on the screen is obtained that is at least good enough to discern single pixels and far in the sub-pixel regime if phase-shifting techniques are used. The principle is sketched in Figure 1.

A major advantage of deflectometry is its very high measurement sensitivity to changes in the slope and the shape of the surface. It can be shown that using standard components from machine vision, it is possible to obtain a slope resolution of tens of $\mu \mathrm{rad}$ or better and, in consequence, a height resolution of tens of nanometers or better $[7,8]$. This sensitivity is made possible by phase shifting and can be enhanced further by increasing the distance between reference pattern and object [2]. Such performance in the range of the wavelength of visible light is especially remarkable, as deflectometry uses only principles from geometrical optics and does not consider any effect of wave optics.

Nowadays, deflectometry is used as an industrial inspection technology for a variety of objects, e.g. for painted surfaces and cast parts in the automobile industry as well as for optical components, such as eyeglass lenses or mirrors in astronomy. However, the applicability of deflectometry is limited to surfaces with a sufficiently high specular reflectivity. This restricts the scope of use, as many industrially produced objects have a diffuse surface during the initial production stages and become specular only later in the process. As the quality of a specular product is assessed by deflectometry on the final product, it would be adequate to use the same technique on the raw object or with an intermediate processing status.

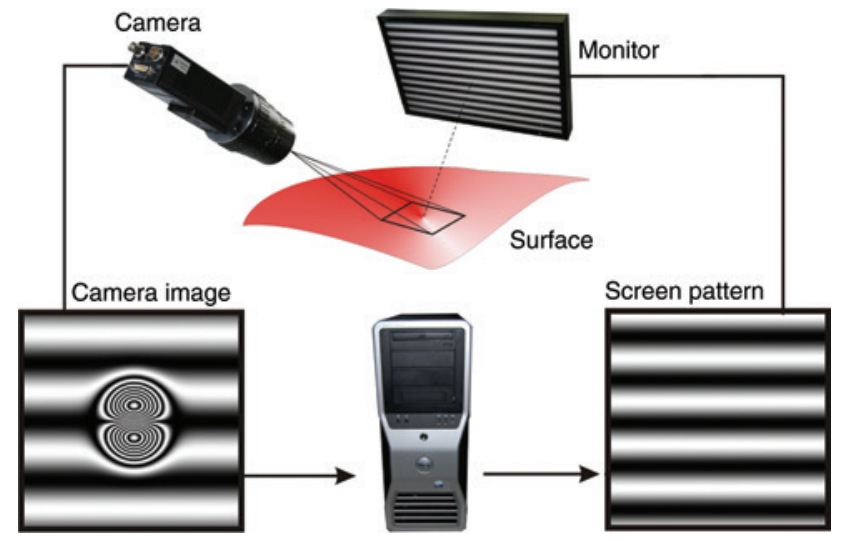

Figure 1: Measurement principle of phase-measuring deflectometry. A series of fringe patterns is displayed as a reference on a screen (e.g. a monitor), and the camera observes the specular reflection of the fringe patterns by the surface under inspection. The shape of the surface directly determines the recorded image. In consequence, distortions of the surface can be detected, and quantitative reconstruction of the surface is feasible.

\subsection{Surface roughness and reflectivity}

The general applicability of deflectometry requires the presence of at least a partially specular reflection at the surface. In addition to the bulk properties of the material, the surface reflectivity is primarily influenced by the roughness of the surface. The roughness is a parameter of the microstructure, which can be a result of a machining process or just the raw state of the material's surface. Such microstructures are usually not resolved by a deflectometry setup, but the roughness, nonetheless, becomes visible by the scattering of light, i.e. a significant proportion of diffuse reflection. This effect can be perceived as a blurring of reflected structures (see Figure 2).

For the application of deflectometry, we want to minimize this undesirable effect (leading to partial or total loss of the reflected-fringe contrast) and maximize the specular part of the reflection. Infrared deflectometry exploits

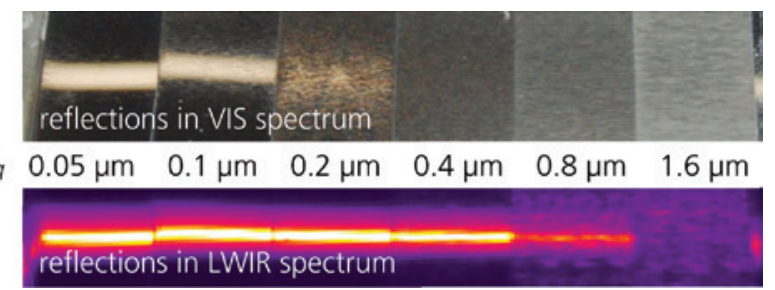

Figure 2: Comparison of wavelength-dependent scattering by calibrated surface-roughness standards in the visible (wavelength $0.4-0.7 \mu \mathrm{m}$ ) and long-wave infrared (wavelength $8-14 \mu \mathrm{m}$ ) ranges. The samples' RMS surface roughness $R_{q}$ is indicated for each area. 
the fact that one of the quantities governing the scattering properties is the wavelength of the reflected light.

A general model for the scattering of electromagnetic waves on rough surfaces was described by Bennett and Porteus in Ref. [17]. We can use a simplified version of this model to quantify the influence factors of this effect. For this, we assume that the incidence of the IR radiation is in surface normal direction, the acceptance angle of the sensor is very small and the height profile of the microstructure has a Gaussian distribution. With these assumptions, the model describes the ratio between the specular reflectivity of the rough surface $\rho$ and the reflectivity $\rho_{0}$ of a perfectly smooth surface of the same material. The simplified model depends only on the wavelength $\lambda$ of the reflected light and on the surface roughness, which is typically quantified by its root-mean-square roughness $R_{q}$ :

$$
\frac{\rho}{\rho_{0}}=e^{-\left(4 \pi R_{q}\right)^{2} / \lambda^{2}}
$$

For the applications considered here, the RMS roughness $R_{q}$ is in the range from tenths of micrometers up to a few micrometers. In this range, the reflectivity for visible light drops significantly. Figure 3 presents a quantitative plot of reflectance vs. $R_{q}$ from 0 up to $1 \mu \mathrm{m}$ for different wavelength ranges. Using light of a longer wavelength in the thermal infrared spectrum, we obtain specular reflection on surfaces, which exhibit a diffuse optical appearance in the visible light spectrum. A thorough study of IR reflectivities was presented in Ref. [18].

The mode of observation is not to be confused with thermography, although similar cameras are used: thermography applications observe the radiation emitted from the surface under inspection, whereas in IR deflectometry, the surface is inspected indirectly using the IR radiation (light) from a dedicated pattern that is reflected at that surface. As such, we do not observe the temperature of the object itself, but we use it as a mirror in which we observe the temperature of the reference screen.

\subsection{Thermal cameras}

For practical applications, the wavelength that can be used is limited by the available technology. The key component here is the camera for the deflectometric sensor. The increase in specularity with wavelength can be demonstrated even for a wavelength increase in the visible spectrum; however, the maximum effect with available technology can be attained in the long wavelength infrared spectrum (LWIR). This part of the spectrum is covered

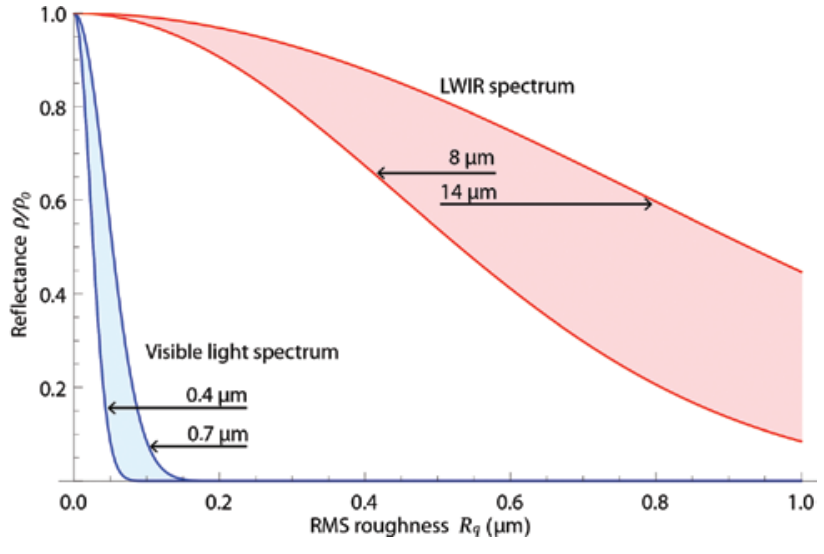

Figure 3: Proportion of specular reflection as a function of $R_{q}$ with wavelengths as parameters, plotted with Eq. (1). The denoted wavelengths give the upper and lower boundaries for the reflectance in the respective wavelength range, the visible range is drawn in light blue, the IR range in light red.

by thermal imaging cameras with either cooled photonic sensors or uncooled microbolometer sensors. While photonic sensors offer higher frame rates and better sensitivity and are available for a larger choice of spectral ranges, microbolometer sensors are commonly used because they are more affordable and offer a slightly wider spectral sensitivity range in the LWIR spectrum $(8-14 \mu \mathrm{m})$.

A future advantage of microbolometer technology is its increased use in applications, like sensors for the automotive sector, which provides for a broader availability of this technology and the prospect of decreasing costs in the future.

A drawback of the thermal infrared spectrum comes with the diffraction limit imposed by the longer wavelength, which limits the maximal achievable resolution of the deflectometry system. The point image is, hence, larger by about an order of magnitude; on the other hand, the pixels of microbolometer cameras are also larger than the pixels for VIS cameras by the same ratio, so that the distinctly lower sensor resolutions (e.g. $640 \times 480$ pixels) are physically well founded and adequate.

Constant improvements in thermal imaging technology in the last years have gradually enabled practical applications for infrared deflectometry. In terms of image resolution and frame rates, the performance of thermal infrared cameras is now almost comparable with conventional cameras [19].

Especially the inspection of raw metal (as flat sheets or molded parts) is an interesting application for IR deflectometry. The roughness of such materials is usually beyond the specular range for the visible spectrum, but well within it for the LWIR spectrum. An additional benefit 
apart from the robustness against surface roughness is that all metals exhibit an increased reflectivity at longer wavelength. Also, IR deflectometry has found applications in optics fabrication, especially aspherics. Large time and cost savings are possible when mirrors can be inspected as mirrors even during the grinding stage [20]. The better the pre-figuring, the less time will be spent polishing with very low material removal rates. Glass can easily be tested with IR deflectometry because the often disturbing rearside reflection [21] is absent: glass becomes opaque in the infrared. This is particularly important as the option of grinding the rear surface for removing the extra reflection would, of course, no longer work.

\section{Thermal patterns}

While the camera technology for a deflectometry sensor is commercially available in the form of thermal IR cameras, there is effectively no suitable technology for generating dynamic thermal patterns in the way a monitor or projector serves the purpose of pattern generation for the visible light spectrum. Recent development in the field of IR deflectometry is, therefore, focused on devising dedicated methods for position encoding with thermal patterns and the evaluation of the acquired heat images.

Formerly, the development of thermal pattern generators was restricted to specialized and very expensive equipment for military applications. The need for hardware test equipment for heat seeking missiles or thermal imagers led to a variety of approaches to simulate dynamic infrared scenes by means of emissive, transmissive, or reflective methods, or with laser scanners. Williams gives an extensive overview of the technologies that emerged from this research in Ref. [22]. Today, the prevailing technology in this field is based on microresistor arrays that create thermal patterns pixel-wise by heating resistors electrically on a chip-scale display.

Unfortunately, apart from being virtually unavailable for civil purposes, these infrared scene simulators are unsuitable for usage in a deflectometry setup. The test equipment usually features only a chip-sized display or a low-power projector directly coupled to the sensor under test. Deflectometry, however, requires a large, ideally planar pattern display. Therefore, in this section, we discuss different pattern-generation methods that are suitable for IR deflectometry.

The challenge in IR deflectometry is to create a spatial and temporal modulation of the thermal pattern with the highest possible contrast. It is fairly easy to create a light source for the LWIR spectrum by means of a heat source, and as the sensitivity of typical IR cameras peaks around room temperature, it can be done safely without high-temperature sources. But heat transfer by conduction, convection, or radiation makes it challenging to maintain cooler areas right next to a heat source to achieve high-contrast patterns. As typical techniques for position coding require a series of different patterns, the necessary temporal modulation of the pattern can complicate things further.

\subsection{Static patterns}

A proven method for the generation of thermal patterns is static patterns. Instead of using regions of different temperature, most methods modulate the emitted infrared radiation by using regions of different emissivity, reflectance, or transmittance. The fact that static patterns cannot be temporally modulated/modified is no exclusion criterion for their use in IR deflectometry. Almost all coding methods for deflectometry use some kind of stripe pattern that is shifted linearly or rotated. The possibility to implement this method with a physically stationary monitor in the visible is simply an addition of convenience in this context, not a necessity.

\subsubsection{Moving emissivity-coded screens}

Figure 4 shows an example for a high-contrast static pattern with differing surface emissivity. While the copper plate is evenly heated to the same temperature, regions with the exposed metal appear cool to the thermal infrared camera due to the near-zero emissivity of the metal. However, the imprinted pattern with significantly higher emissivity shows the actual temperature of the surface.

Of course, temporally varying patterns can also be created and recorded by mechanically moving a static pattern. This method was first used by Kammel and Horbach in Ref. [23] to implement position encoding with a single phase-shifted binary pattern, which demonstrated for the first time the applicability of IR deflectometry on unpainted car body parts. Surface defects were detected with a simple evaluation based on the contrast and modulation information of the phase-shifted pattern. Figure 5 shows an embodiment of the technique.

In a similar experiment in Ref. [24], a full phase-shift code sequence was obtained by shifting and rotating multiple static sine patterns. In this case, the application was the surface inspection of transparent materials that become opaque in the far infrared spectrum, so that artifacts from multiple reflections can be avoided. 

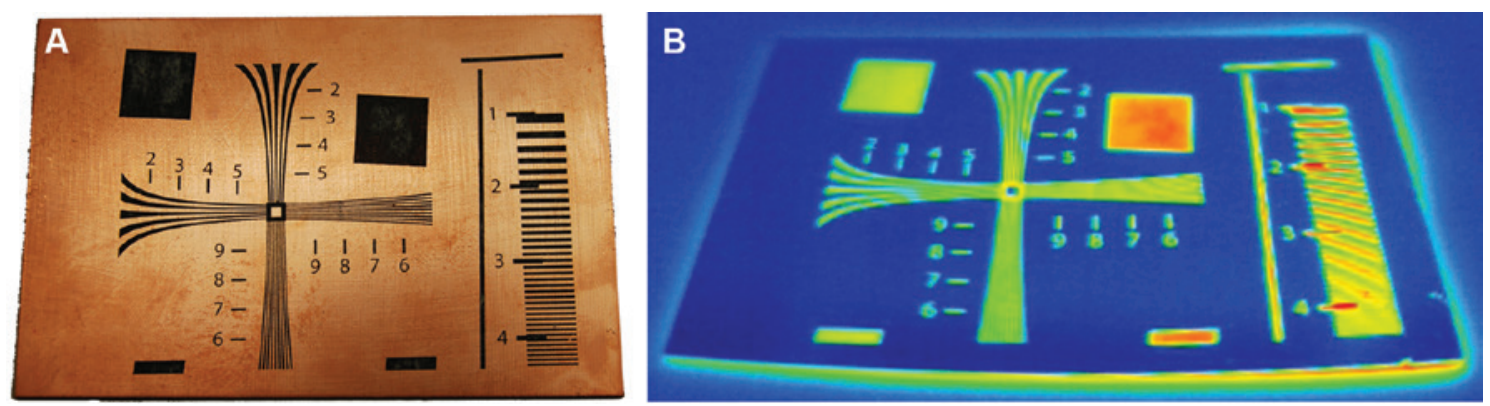

Figure 4: Example for a high-contrast static pattern with differing surface emissivity. The object (dimensions $120 \mathrm{~mm} \times 80 \mathrm{~mm}$ ) is a blank for printed circuit board production, the surface is brushed copper, and the patterns consist of transferred laser-printer toner. (A) Appearance in visible light, (B) false-color image of plate (heated to $80 \mathrm{~K}$ over room temperature) in thermal IR; blue means low radiation, red means high radiation.

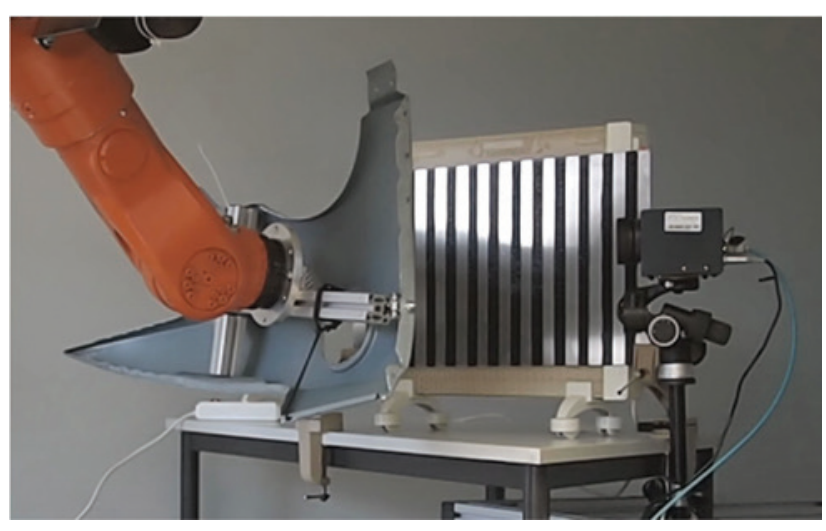

Figure 5: Laboratory setup for testing a car body part with fixed pattern that is translated in steps of a quarter of its period to implement a simple phase-shifting method. The low-emissivity carrier metal is aluminum in this case.

A disadvantage of this approach is that care has to be taken where the reflection of the object is directed. If the measurement setup is not designed carefully, heat sources in the surroundings, such as the camera itself, end up as ghost images superimposing the images taken by the IR camera.

\subsubsection{Radiating hot wire}

Another successful approach with static patterns was demonstrated in the inspection of unpolished astronomical telescope mirrors during the grinding stage [20], where a scanning hot wire is used as an IR line light source. To implement the position encoding, the wire is moved with a motorized stage horizontally and vertically. The wire as IR light source has the advantage that it can be suspended in free air and heated to high temperatures in order to create a high-contrast pattern in the IR spectrum. At the same time, a single-line pattern entails the disadvantage that many frames have to be acquired to sample the whole surface, so that the frame rate of the camera sets a lower boundary for the total measurement time.

To obtain position coding with this approach, the scanned line pattern has to be located accurately in the camera images. A robust method is to calculate the centroid of the intensity for each pixel over a sequence of images [25]. If the movement of the line pattern is slow enough, each camera pixel takes multiple intensity measurements of the passing line profile from which the position of its center can be obtained. This gives an instant of maximum intensity for each pixel and, given the corresponding position of the line pattern, a position encoding between camera and pattern.

The heated-wire concept was later adapted for the inspection of arbitrary surfaces in a scanning sensor setup [26]. Here, the movement is restricted to only one direction, and only partial position coding is obtained, but at the same time, the scanning approach enables the inspection of large-scale surfaces or inline inspection of linearly moving objects. It was also shown that for fastmoving line patterns, e.g. at a setup for inline inspection, or on surfaces that distort the reflected pattern significantly, an actual line detection (as opposed to centroiding) can obtain better results. A new tensorbased method was introduced in Ref. [26] that performs a subpixel-accurate line detection for each image and interpolates the movement of the pattern between single frames.

This method was used with the setups shown in Figure 6, also in connection with a heated wire as pattern source. Figure $6 \mathrm{~A}$ shows the concept as a robot-mounted version of the sensor, which provides a flexible way to inspect large-scale surfaces of arbitrary geometry by scanning them in a continuous path. The reverse concept is 

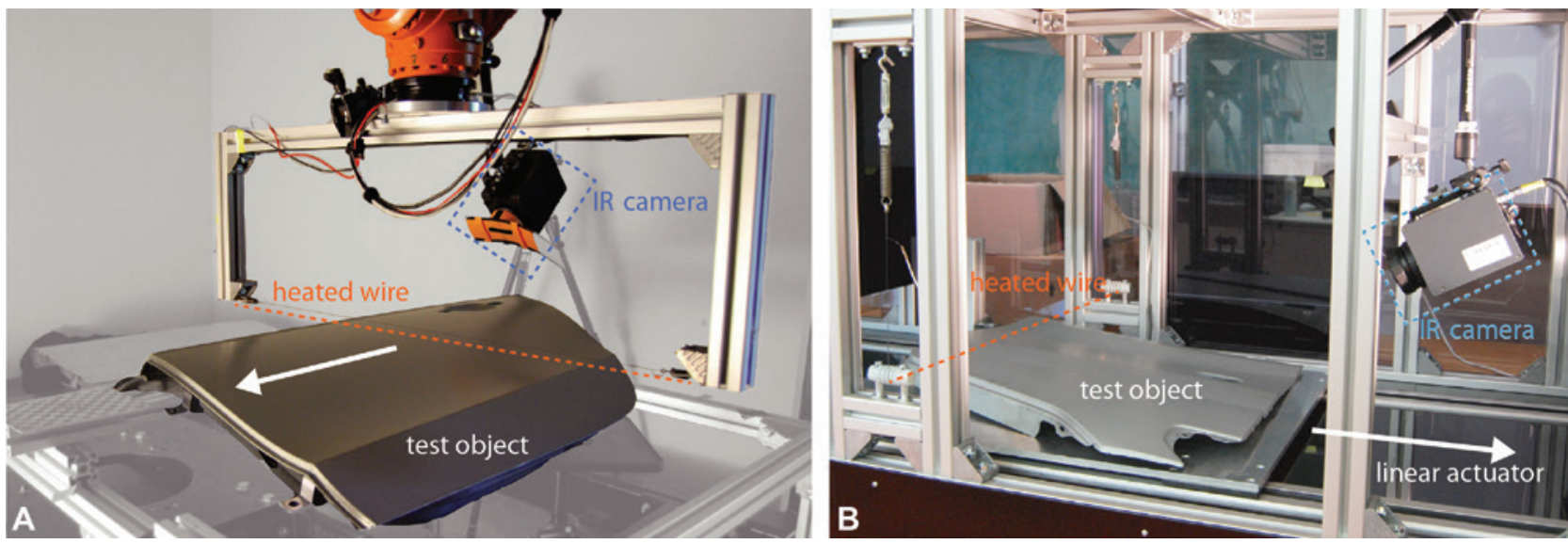

Figure 6: Two setups that use a single heated wire as pattern source to linearly scan the surface under inspection. (A) Robot-mounted sensor that can be moved in arbitrary paths over the surface and enables the inspection of large-scale surfaces; (B) setup for inline inspection where the object is moving and the sensor is mounted in a fixed configuration over the transport mechanism.

shown in Figure 6B where the object to be inspected is moving, e.g. on a conveyor belt, and is inspected while passing under the fixed mounted sensor.

\subsection{Dynamic patterns}

Another approach for realizing position encoding is to create planar thermal patterns dynamically, as a display screen for the thermal infrared spectrum, so to speak. As previously mentioned, the difficulty lies in creating and maintaining a high temperature difference in a small space, which is necessary for a high-contrast pattern.

Unfortunately, the existing approaches to generate static patterns in IR deflectometry, e.g. modulating emissive, reflective, or transmissive material properties, cannot be applied to generate dynamic patterns. Common methods for dynamic patterns from the visible spectrum, like LED, LCD, or CRT technologies, are also not viable for the thermal IR spectrum [27] or, in case of digital micromirror devices, would need extensive redesign to be applicable [22].

For a display based on regions with a different temperature, heat diffusion leads to fading of the pattern and cross-talk between neighboring regions. Moreover, the energy required to change the displayed pattern increases significantly with the size of the display area as it has to be heated up or cooled down. Therefore, such displays need careful design.

For IR deflectometry, two new methods for creating dynamic patterns have been tested by the authors: pattern generation with large-scale resistor arrays (as opposed to specialized arrays with pixel pitches of several tens of $\mu \mathrm{m}$
[28] and luminosities insufficient for deflectometry) and surface heating with laser radiation.

\subsubsection{Resistor arrays}

Displays based on resistor arrays use the heat dissipation in resistors as IR source. By arranging the resistors in a grid pattern and controlling the electrical current for each resistor individually, a display for the IR spectrum can be realized that allows the display of arbitrary pixel images. This method was already proposed in Ref. [27] and implemented on a small scale in Ref. [24].

Figure 7 shows a resistor array setup and the resulting image in the LWIR spectrum. Tiling such arrays makes displays of arbitrary size possible. The challenges of this design lie in the practical implementation. Problems like the heat transfer through the base material, and cooling of the display when pixels are switched off, have to be solved, and a glance at the aforementioned microresistor arrays shows that this requires extensive engineering efforts even at small scales. While resistor arrays are definitely a viable technology for application in IR deflectometry, their design and operation becomes complex for higher frame rates and pixel densities.

\subsubsection{Laser-heated screens}

Another approach is to use laser heating for creating thermal IR displays, which has been demonstrated in Ref. [29]. By using a powerful laser with a mirror scanner, regions on a projection surface can be heated locally with 


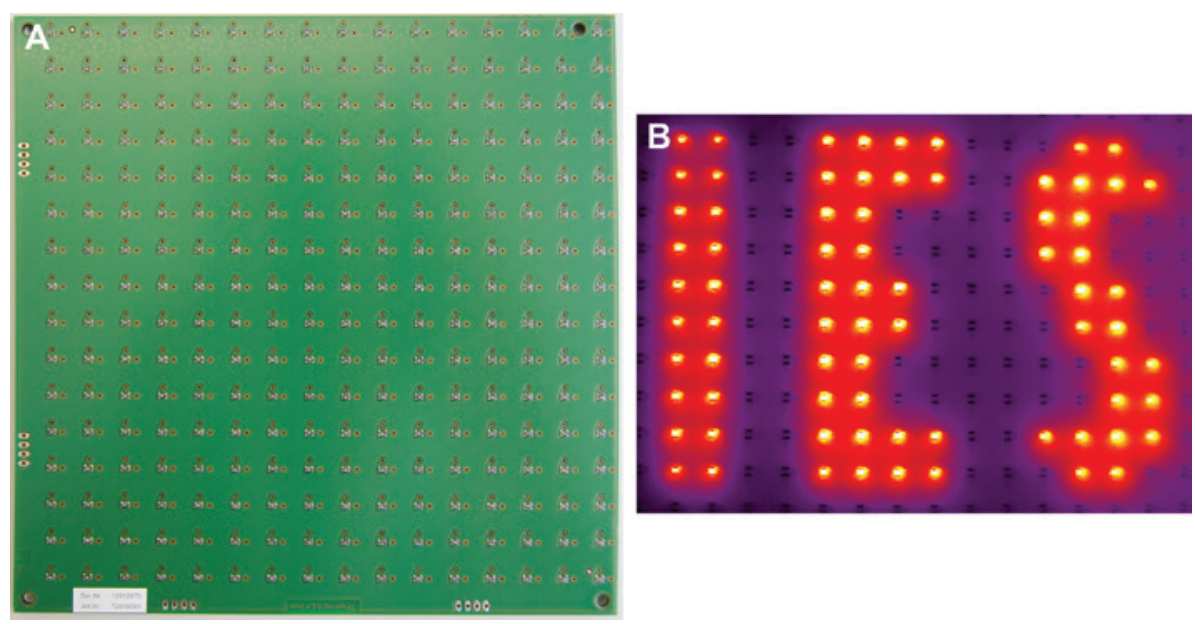

Figure 7: Resistor array for thermal pattern generation. (A) Prototype circuit board with a $16 \times 16$ array of SMD resistors. (B) By controlling the current flowing through each resistor individually, arbitrary pixel images can be displayed.

the aim that the resulting heat signature represents the desired pattern. This way, off-the-shelf laser hardware can be used to create large-scale thermal patterns. Unlike conventional projections, the laser light is mostly absorbed and heats the surface instead of being scattered. Still, there must be no direct reflections of the laser beam into the IR camera, as the intensity is high enough to destroy the camera sensor.

The drawback of this method is that the pattern buildup is not instantaneous. Already written parts of the pattern slowly diffuse and cool down while the laser is still working.

Figure $8 \mathrm{~A}$ shows a laboratory setup with a conventional laser system as used for laser processing, where the laser beam is steered by a mirror scanner. The projection screen takes the role of the pattern display, and the setup with the camera observing the reflection of the pattern in the surface under test represents a deflectometry setup. Figure 8B shows several snapshots of the resulting pattern on the screen, where the current position of the laser spot is clearly visible. To achieve position coding with such a fading pattern, the camera records a video sequence during the writing process. From the current position of the laser spot in each frame of the sequence and the corresponding known position of the laser on the projection screen, a rough position encoding can be obtained. This can be extended with a model of the projection surface's thermodynamic behavior to interpolate the laser position even between single frames and get a more precise position decoding. Following the time arrow in Figure 8B allows building accurate cooling curves. Eventually, a complete pattern can be built up from all recorded frames as in Figure 8C.
The advantage of this method is that it can easily be scaled up to large projection surfaces, but at the cost of longer build up times for the pattern. This could be compensated by increasing the laser's power output to maintain the pattern contrast at higher write speeds.

\section{Applications}

\subsection{Sensitive defect detection on visually diffuse objects}

The target application for most developments in IR deflectometry is the inspection of objects with raw metal surfaces in the steel or automotive industry. While there are many options to perform IR deflectometry with the previously shown methods, a lightweight and mobile setup is preferable, which is why the scanning setup from Figure $6 \mathrm{~A}$ has proven to be the most versatile one for this application. Figure 9 shows the result from scanning a car body sheet as a fused image from multiple scan lines (line pitch in scan direction: $2 \mathrm{~mm}$ ) and the derived local gradients of the surface. The surface has been scanned vertically and horizontally to obtain both gradients, but only one fringe image is shown here.

The defects in Figure 9A are usually only visible by manual inspection after honing the surface with a dressing stone. Not so in deflectometry: defects appear as minima and maxima in the gradient image and can easily be detected. The actual depth of the defects ranges from $50 \mu \mathrm{m}$ to $400 \mu \mathrm{m}$, as determined both by the integration 

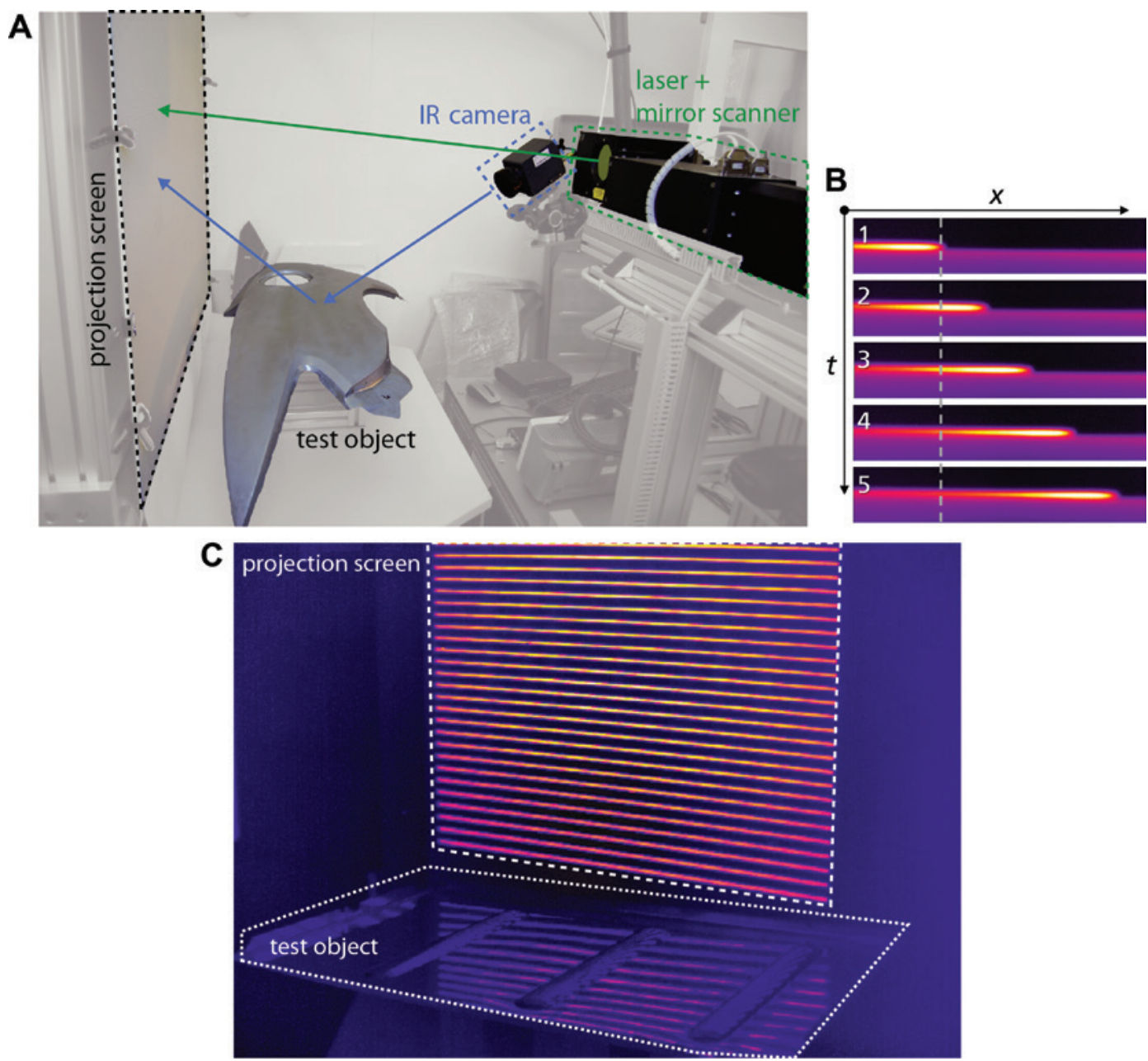

Figure 8: Thermal pattern generation with a laser. An off-the-shelf laser processing system is used to inscribe heat patterns onto a projection surface (A). (B) Decay of thermal pattern as the laser beam is moved across the screen. In this example, the laser's scan speed was $40 \mathrm{~mm}$ per frame. The numbers in the individual frames give the frame count; note the quick cooling at a given location (indicated by dashed line). (C) Complete thermal pattern, synthesized from all recorded frames. With 100-W output power, it takes about $8 \mathrm{~s}$ to write the pattern shown with a temperature contrast of $12 \mathrm{~K}$. For comparison, the temperature equivalent noise of the camera sensor is about 50 mK RMS.
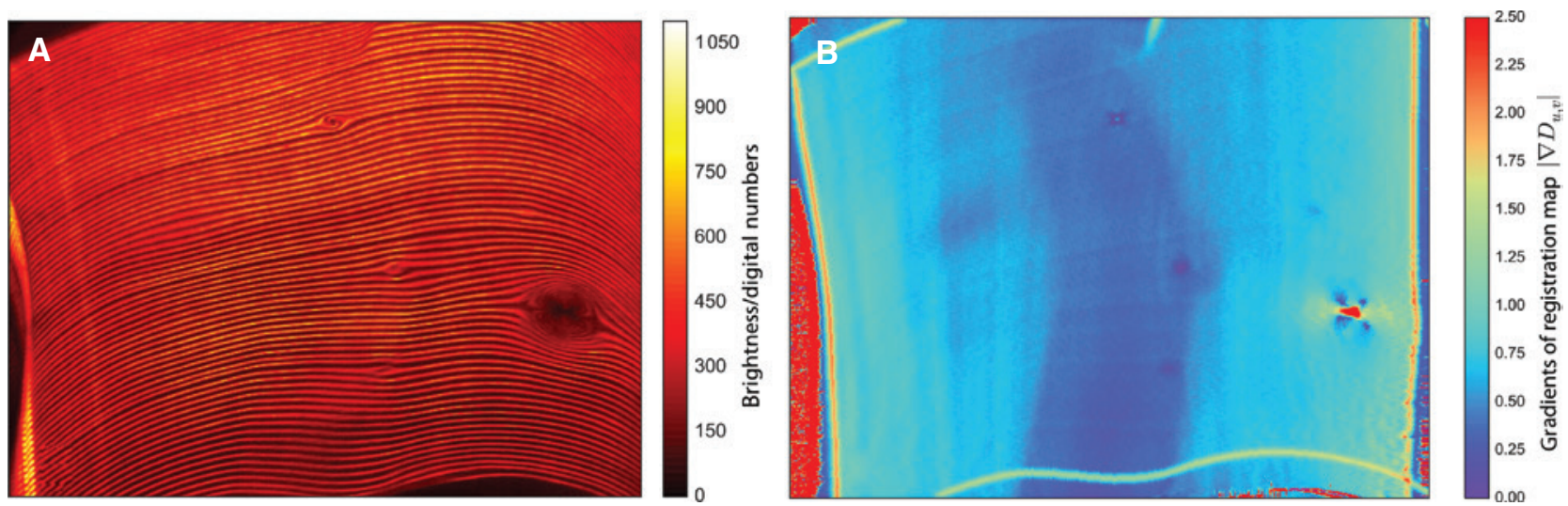

Figure 9: Results from a car body sheet measured with a scanning sensor. The images show a 300-mm $\times 250 \mathrm{~mm}$ section of the surface. (A) Vertical-scan lines merged into a single image, defects are readily visible as distortions; (B) computed local gradients, where defects are clearly visible as minima or maxima in the gradient map. 


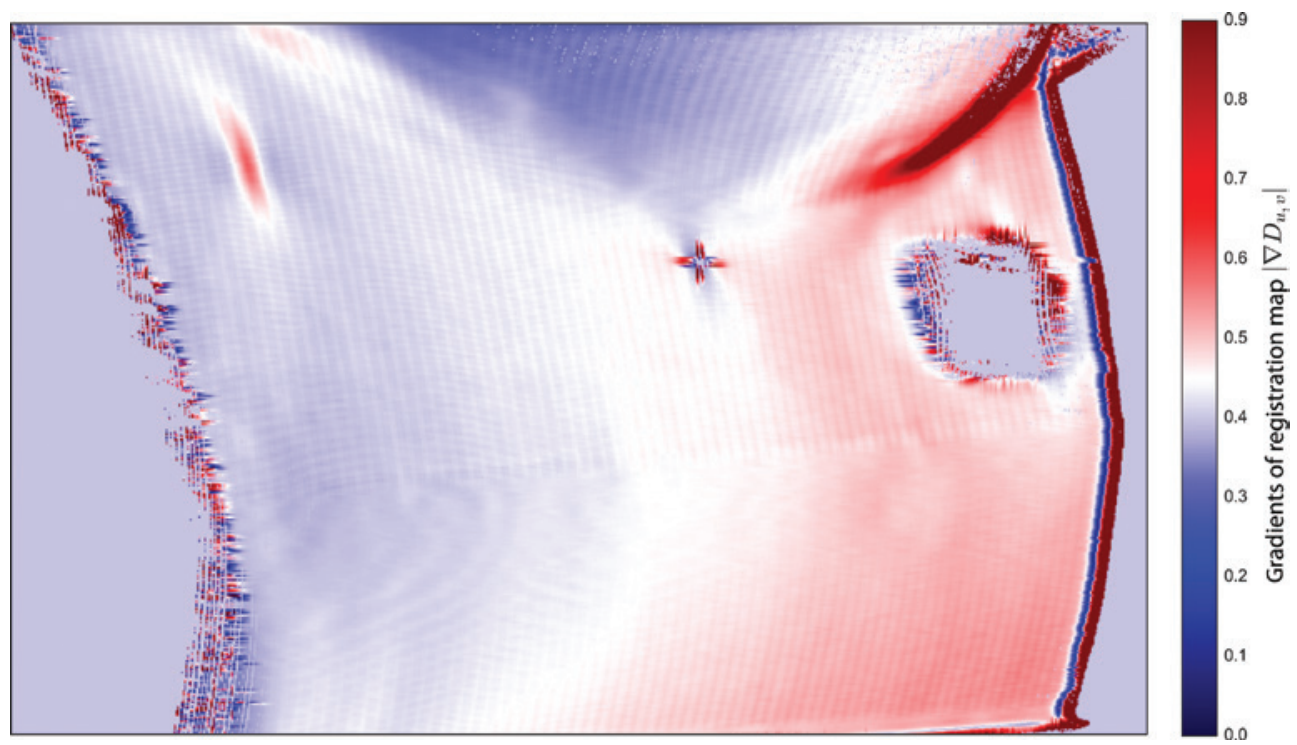

Figure 10: Gradient map of a car body part resulting from measurement during linear movement of the part. The part was moving from left to right under the sensor, which was fixed over the transport mechanism.

of the deflectometric gradient maps and cross-checks with a focusing microscope.

Another possible setup for a scanning IR deflectometry sensor is to use it for inline inspection where the objects to be inspected are already moving, e.g. on a conveyor belt during manufacturing. The objects are inspected while passing the sensor, provided the surface geometry is accessible from a static position of the camera. A result that was acquired with the scanning setup from Figure 6B on a predominantly flat car body part with a raw metal surface is shown in Figure 10. The image shows the local change in the measured gradients where surface defects, such as dents in the material, and edges in the geometry stand out as sudden changes.

When custom building the pattern generators cannot be avoided, one weakness of IR deflectometry (not having programmable off-the-shelf display screens available) turns into a strength: the pattern generators can be made to the specifications of the task, including for dedicated surface shapes and/or large object sizes.

\subsection{Optics figure measurements in preliminary processing stages}

Besides the benefit of IR deflectometry to enable measurements of visually rough surfaces, an additional advantage materializes in the surface inspection of transparent parts. Materials like glass and many transparent plastics are nearly completely transmissive in the visible light spectrum, but become opaque in the LWIR spectrum (which is why IR imaging optics use Germanium lenses, being opaque in the visible but transparent in the IR). As referenced above in Refs. [20, 25], it is, therefore, possible to measure glass optics that is ground on both sides but still reflective in the IR. Whether the tested surfaces are ground or polished, the parasitic rear-side reflection does not occur. One example of this is shown in Figure 11.

In Figure 11A, front and rear side reflection patterns are visible, and scratched/scuffed areas in the center stand out clearly. In Figure 11B, only the front-side reflection appears; the scuffed areas are still visible, which means that their depth is a significant proportion of the IR wavelength. Also, the IR image is less sharp because of the reduced resolution due to both wavelength and pixel resolution of IR cameras, which was discussed above.

\section{Summary}

Current developments in IR deflectometry show that it has grown from first proofs of concept to a viable method for surface inspection. Especially its applicability on surfaces that are difficult to inspect, such as diffusely specular surfaces or materials that are transparent in the visible spectrum, makes it stand out among other optical inspection methods. The highest demand for IR deflectometry is currently for the inspection of raw metal surfaces primarily in the car industry. However, other applications already realized, like the inspection of telescope mirrors in early production stages, show that there are many fields where 

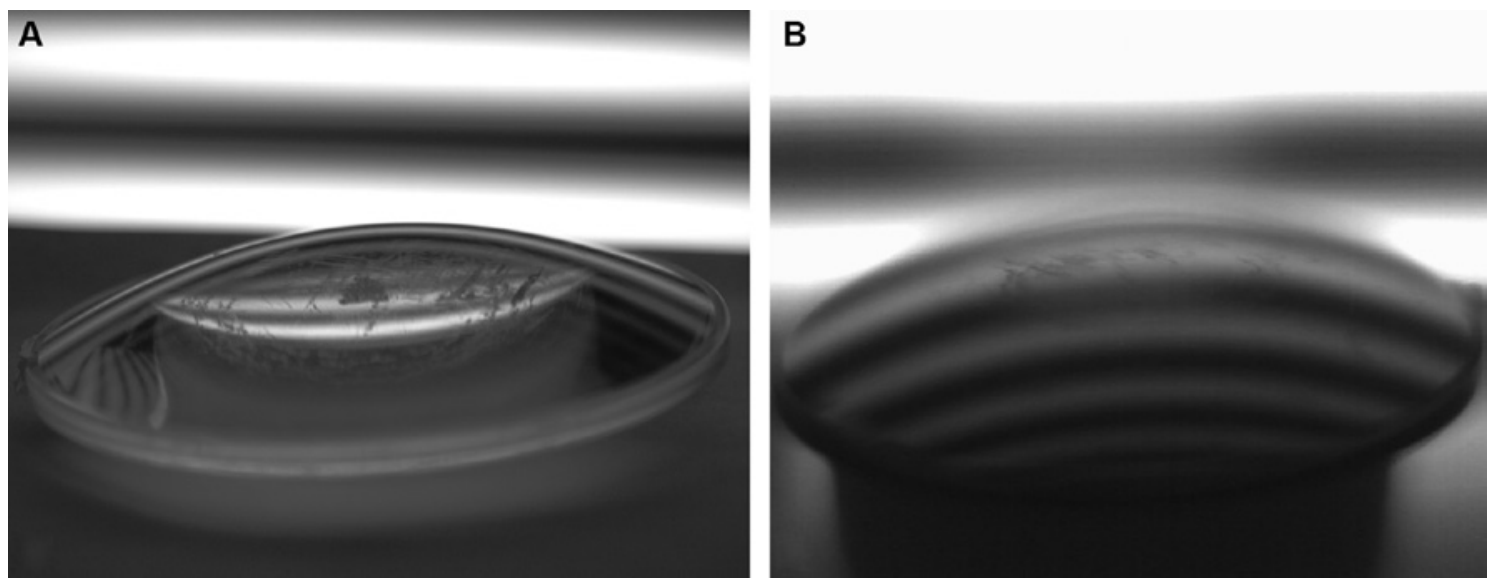

Figure 11: Fringe reflections from the same plastic lens in the visible (A) and IR (B).

the particular advantages of IR deflectometry make it uniquely suitable for the task.

IR deflectometry enables the application of established methods and algorithms from conventional (visible spectrum) deflectometry to broaden the range of surfaces and materials that can be tested. Even though the generation of thermal patterns currently enforces significantly different setup concepts for thermal-infrared deflectometric sensors, the developments have led to new ways of doing deflectometry, like the scanning of surfaces, which enables new and relevant applications.

\section{References}

[1] S. Zhang, in 'High-Speed 3D Imaging with Digital Fringe Projection Techniques' (CRC Press, London, 2016).

[2] J. Beyerer and D. Pérard, Tech. Mess. 64, 394-400 (1997).

[3] R. Höfling, P. Aswendt and R. Neugebauer, Opt. Eng. 39, 175-182 (2000).

[4] M. Petz, Tech. Mess. 71, 389-397 (2004).

[5] J. Kaminski, S. Lowitzsch, M. C. Knauer and G. Häusler, in 'Fringe 2005', Ed. by W. Osten (Springer, Heidelberg, 2005) pp. 372-379.

[6] M. C. Knauer, Absolute Phasenmessende Deflektometrie, Dissertation, Institute of Optics, Information and Photonics, Max Planck Research Group, Friedrich-Alexander-Universität Erlangen-Nürnberg (2006).

[7] P. Su, R. E. Parks, L. Wang, R. P. Angel and J. H. Burge, Appl. Opt. 49, 4404-4412 (2010).

[8] S. B. Werling, Deflektometrie zur automatischen Sichtprüfung und Rekonstruktion spiegelnder Oberflächen, Dissertation, Lehrstuhl für Interaktive Echtzeitsysteme am KIT, Fraunhofer IOSB (KIT Scientific Publishing, Karlsruhe, 2011).

[9] W. Li, T. Bothe, C. von Kopylow and W. Jüptner, in 'Optical Metrology in Production Engineering', Ed. by W. Osten and M. Takeda, Proc. SPIE 5457 (SPIE, Bellingham, 2004) pp. 300-311.
[10] J. Balzer and S. B. Werling, Tech. Mess. 74, 545-552 (2007).

[11] S. Ettl, J. Kaminski, M. Knauer and G. Häusler, Appl. Opt. 47, 2091-2097 (2008).

[12] V. M. Patel and R. Chellappa, in 'Excursions in Harmonic Analysis Volume 1' (Birkhäuser, Boston, 2013) pp. 377-398.

[13] L. Huang, M. Idir, C. Zuo, K. Kaznatcheev, L. Zhou, et al., Opt. Laser Eng. 64, 1-11 (2015).

[14] J. Burke, in 'Optical Methods for Solid Mechanics' (Wiley-VCH, Weinheim, 2012) pp. 83-139.

[15] W. Nadeborn, W. Osten and P. Andrä, Opt. Laser Eng. 24, 245-260 (1996).

[16] K. Falaggis, D. P. Towers and C. E. Towers, in '22nd Congress of the International Commission for Optics: Light for the Development of the World', Ed. by R. Rodríguez-Vera and R. Díaz-Uribe, Proc. SPIE 8011 (SPIE, Bellingham, 2011), paper 80117H.

[17] H. E. Bennett and J. O. Porteus, J. Opt. Soc. Am. 51, 123-129 (1961).

[18] Z. Sárosi, W. Knapp, A. Kunz and K. Wegener, in 'InfraMation 2010 Proceedings’, Infrared Training Center (2010).

[19] H. Budzier and G. Gerlach, Thermische Infrarotsensoren, Grundlagen für Anwender (Wiley-VCH, Weinheim, 2010).

[20] T. Su, S. Wang, R. E. Parks, P. Su and J. H. Burge, Appl. Opt. 52, 7117-7126 (2013).

[21] C. Faber, M. C. Knauer and G. Häusler, in 'Proceedings of the 110th Annual DGaO Conference', A10 (2009).

[22] O. M. Williams, Infrared Phys. Technol. 39, 473-486 (1998).

[23] J. W. Horbach and S. Kammel, in 'Machine Vision Applications in Industrial Inspection XIII', Ed. by J. R. Price and F. Meriaudeau, Proc. SPIE 5679 (SPIE, Bellingham, 2005) pp. 108-117.

[24] S. Höfer, M. Roschani, S. B. Werling and J. Beyerer, in 'Tagungsband des XXV. Messtechnischen Symposiums des AHMT', Ed. by F. Puente León and J. Beyerer (Shaker, Aachen, 2011) pp. 127-138.

[25] T. Su, W. H. Park, R. E. Parks, P. Su and J. H. Burge, in 'Optical Manufacturing and Testing IX', Ed. by J. H. Burge, O. W. Fähnle and R. Williamson, Proc. SPIE 8126 (SPIE, Bellingham, 2011), paper $81260 \mathrm{E}$.

[26] S. Höfer and J. Beyerer, Tech. Mess. 83, 374-385 (2016).

[27] Z. Sárosi, W. Knapp, A. Kunz and K. Wegener, in 'InfraMation 2010 Proceedings', Infrared Training Center, http:// 
www.inspire.ethz.ch/ConfiguratorjM/publications/Detection_128316497984543/One_shot_deflectometry_in_IR_ v4.pdf (2010).

[28] O. M. Williams, G. C. Goldsmith II and R. G. Stockbridge, in 'Technologies for Synthetic Environments: Hardware-in-theLoop Testing X', Ed. by R. L. Murrer Jr., Proc. SPIE 5785 (2005) pp. 208-224.

[29] S. Höfer, S. B. Werling and J. Beyerer, in 'Proceedings SENSOR 2013’ (2013) pp. 785-790.

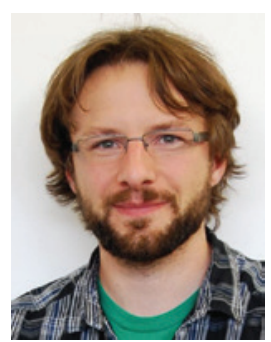

\section{Sebastian Höfer}

Fraunhofer Institute of Optronics, System Technologies and Image Exploitation Systems for Measurement, Control and Diagnosis (MRD), Karlsruhe, DE 76131 Fraunhoferstraße 1, Germany

Sebastian Höfer worked as a PhD student in Computer Science at the Karlsruhe Institute of Technology on deflectometry in the thermal infrared spectrum and finished his thesis and exam in 2016. Since 2015, he has also been a scientific consultant at the Fraunhofer IOSB in Karlsruhe. His fields of research include (infrared) deflectometry, thermography, and computer vision systems.

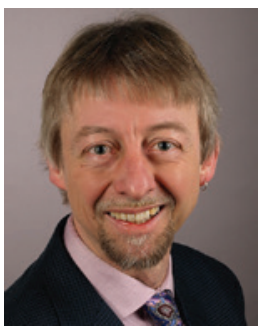

Jan Burke

Fraunhofer Institute of Optronics, System Technologies and Image Exploitation Systems for Measurement, Control and Diagnosis (MRD), Karlsruhe, DE 76131 Fraunhoferstraße 1, Germany Jan.Burke@IOSB.Fraunhofer.de

Jan Burke holds a PhD in Physics from the University of Oldenburg, Germany, and has since worked on a wide variety of topics in optical metrology in California, Australia, and most recently, Bremen, where he made the transition from interferometry to deflectometry, in search for better and cheaper asphere and free-form measurement technologies. Since 2016, he has been with the Fraunhofer IOSB as a research group leader. His main research interests are in fringe processing and low-uncertainty optical measurements.

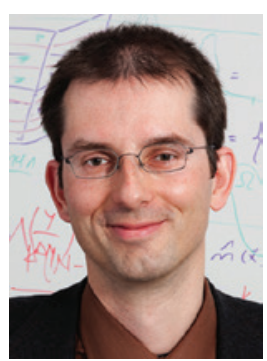

\section{Michael Heizmann}

Karlsruher Institut für Technologie (KIT) - Institute of Industrial Information Technology, Hertzstraße 16, Geb. 06.35 Karlsruhe 76187, Germany

Michael Heizmann is Professor and Director of Institute at the Institute of Industrial Information Technology (IIIT) at the Karlsruhe Institute of Technology (KIT) and Scientific Advisor at the Fraunhofer Institute of Optronics, System Technologies and Image Exploitation IOSB in Karlsruhe, Germany. His research areas include machine vision and image processing, image and information fusion, metrology, automation, and their applications in industry. 